\title{
San Miguel y el oriente salvadoreño. La construcción del Estado de El Salvador, 1780-1865
}

\author{
Armando Méndez Zárate (D) http://orcid.org/0000-0003-0586-5807 \\ Centro de Investigaciones Multidisciplinarias sobre Chiapas y la Frontera Sur \\ Universidad Nacional Autónoma de México, México \\ armando.mendez.zarate@gmail.com
}

Clara Pérez Fabregat, San Miguel y el oriente salvadoreño. La construcción del Estado de El Salvador, 1780-1865, San Salvador, UCA Editores, 2018, 452 p., ISBN: 978-99961-1-052-8.

El texto de Clara Pérez Fabregat es fundamental para entender la construcción del Estado en Centroamérica durante el siglo XIX. El análisis de los fenómenos económicos y políticos del oriente salvadoreño, nos acerca a las dinámicas locales que influyeron en la reconfiguración territorial de El Salvador y el surgimiento de actores políticos con preeminencia más allá de las fronteras políticas externas e internas reconocidas en Centroamérica. Además, de ofrecer otros puntos de partida para dialogar con los estudios históricos decimonónicos sobre México y América Latina desde una perspectiva comparativa. Toda vez que el método empleado por la autora permite establecer líneas de comparación con otros territorios y procesos históricos.

La investigación de Clara Pérez Fabregat fue organizada en nueve capítulos y es resultado de la tesis doctoral en historia de la autora. El estudio fue parte del proyecto State Building del European Research Council dirigido por Juan Carlos Garavaglia y Claudia (c) (7) (8) No-Comercial 4.0 Internacional.

Secuencia. E-ISSN 2395-8464 http://secuencia.mora.edu.mx/

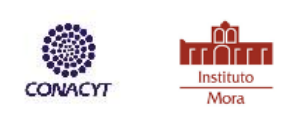


Contente en la Universidad Pompeu Fabra. En este contexto, la revisión histórica sobre el oriente salvadoreño, permite observar críticamente, las coyunturas políticas, económicas y sociales de esta parte de América. De esta forma, la investigación revitaliza la historiografía salvadoreña por su aporte metodológico y su discusión teórica sobre los procesos políticos, sociales y económicos en El Salvador durante el siglo XIX.

La autora utilizó un método de análisis histórico basado en la consulta exhaustiva de archivos, configurando un texto dividido en dos partes: en la primera muestra un panorama social y económico de la "región salvadoreña” (p. 30) desde finales de la época colonial hasta los primeros años del estado federal de El Salvador (1824-1841). La segunda parte utiliza un análisis fino para describir la forma en la que las elites locales del oriente se apropiaron de los puestos de gobierno nacional y controlaron la economía regional, apalancadas por el control de los recursos ante los conflictos armados que legitimaron y afianzaban su poder, hasta su declive a partir de 1865.

En los capítulos de Pérez Fabregat resalta un tratamiento histórico del desorden y la violencia del siglo XIX en El Salvador, como "consecuencia de las luchas entre distintos actores sociales por el acceso y control de los escasísimos recursos económicos” (p. 296). Esta hipótesis ayuda a reflexionar y dimensionar el uso de la fuerza empleada por los políticos, militares y comerciantes en El Salvador y Centroamérica para alcanzar sus objetivos políticos, de ahí la explicación de la inestabilidad crónica del siglo XIX sufrida por los países centroamericanos.

Esta interpretación de Pérez Fabregat fue posible gracias a la utilización de las fuentes históricas (documentos, informes, cartas, periódicos) que fueron consultados en fondos documentales y archivos de Europa y Centroamérica. El aporte a la historia regional de Centroamérica, llama la atención por las particularidades del oriente salvadoreño, comparado con el occidente y el centro de El Salvador, donde ocurrieron otras dinámicas económicas, Secuencia. E-ISSN 2395-8464 
sociales y políticas. Esto a pesar de estar en un territorio pequeño pero estratégico en el contexto centroamericano.

Este último punto, me remite a resaltar la relevancia de El Salvador como centro y escenario de los movimientos políticos en Centroamérica en el siglo XIX. Sin embargo, pese a ese protagonismo, El Salvador tuvo inconclusa la construcción de sus instituciones gubernamentales nacionales (poderes ejecutivo, judicial, legislativo). Además, de tratar de instaurar un sistema fiscal adecuado a las demandas del gobierno nacional que financiara los conflictos armados en los cuales se involucraba El Salvador.

Otros aspectos fundamentales de la obra resultan de los aportes revisionistas de las teorías y métodos que explican los desequilibrios económicos y los contrastes sociales en El Salvador, particularmente aquellos vinculados con el modelo agroexportador y cafetalero de finales del siglo XIX. Al respecto, Pérez Fabregat retoma una propuesta interesante al reconsiderar la geografía como elemento clave en la construcción de los Estados nación en Centroamérica. En este sentido, recalca la consideración de los elementos naturales (ríos, llanuras, montañas, costas) y el territorio como actores centrales y no como una unidad administrativa creada por factores arbitrarios.

Por ejemplo, el río Lempa resalta como un actor de primer orden para la historia salvadoreña (p. 28-29), revalorizando los atributos geográficos como factores decisivos en la formación y consolidación de los procesos históricos de El Salvador y Centroamérica. Al respecto, resultaría interesante que alguna línea de investigación desde la historia ambiental estime el río Lempa como el eje articulador de la geografía e historia salvadoreña al dividir físicamente al país y los procesos históricos que ocurrieron en ambas márgenes del río. Además, aportaría un método más para la historia salvadoreña y centroamericana, pues permitiría renovar lo que conocemos a través de las fuentes documentales disponibles en los Secuencia. E-ISSN 2395-8464 
archivos y bibliotecas de El Salvador y, revisitar otros procesos y coyunturas empleando los recursos naturales como fuente histórica.

El interés por la historia ambiental subyace entre líneas en la obra de Pérez Fabregat, pero no dedica un capítulo especial a discutirlo, debido al enfoque político y fiscal de su investigación. No obstante, esta herramienta le permitió subsanar carencias documentales y lagunas de información, pues uno de los problemas para hacer historia en El Salvador radica en la falta de documentación seriada y completa de fondos (documentos, informes, cartas, periódicos) que den cuenta y noticia de periodos prolongados.

El problema de las fuentes seriadas para la construcción de una historia fiscal y política salvadoreña fue más evidente en el texto, cuando la autora explicó la fiscalidad, el gasto y la distribución de los recursos del Estado durante la primera mitad del siglo XIX. A pesar de estas limitantes metodológicas, Pérez Fabregat logró elaborar un estudio interesante sobre el gasto, la obtención de recursos, la adquisición y pagos de deudas contraídas por el Estado e identificar a los acreedores de esas deudas y el uso que se le dieron a esos recursos.

Aunque la autora resolvió las lagunas documentales con una extensa revisión de los fondos del Archivo General de la Nación de El Salvador (AGNES). En los capítulos se demuestra la consulta minuciosa de los fondos del AGNES, además de aportarnos datos abundantes rescatados de las cartas, periódicos, diarios, informes, proclamas y correspondencia. La información de las referencias abre una ventana que nos permite observar la riqueza documental del AGNES, especialmente el Fondo de Quemados que constituye el grueso de la información utilizada por Pérez Fabregat. Incluso se distinguen otras temáticas que pueden ser analizadas por la historia fiscal, política y regional empleando los mismos documentos. 
Otro de los problemas reiterativos en la obra de Pérez Fabregat consistió en la imposibilidad de ofrecer conclusiones más concretas de los procesos económicos, fiscales y políticos regionales del oriente salvadoreño y demostrar el impacto sobre el resto de El Salvador. En primer lugar, por la problemática de las fuentes anteriormente expuestas y que son reconocidos por la autora en el texto. Además, de que se requeriría un esfuerzo más amplio de consulta en otros archivos de Centroamérica, Estados Unidos de América y Europa para poder completar esas series, con sus respectivos costos humanos y financieros que implicaría una investigación de esa envergadura.

No obstante, la autora avanzó en el proceso de interpretación y análisis de fuentes, incorporando la información disponible y nos presentó un buen ejercicio de análisis histórico. Especialmente, cuando aborda la crisis del endeudamiento y la fiscalidad de los gobiernos salvadoreños, el cual es un tema que resalta en la mayoría de los capítulos, aunque de forma más puntual en la primera parte de la obra. Este ejercicio de análisis pudiera ser replicado en otros territorios contiguos como Nicaragua, Honduras y Guatemala.

De manera crítica considero que existe una repetición de los contenidos, en especial en aquellos capítulos donde realiza un contexto histórico sobre la construcción del Estado nacional salvadoreño a partir de 1821. Esto también pudiera ser una ventaja, ya que se puede retomar la lectura de la obra al inicio de las dos partes, sin necesidad de haber leído de forma continua todos los capítulos. Además ofrece un buen material de introducción para los lectores que no están relacionados con la historia centroamericana y salvadoreña.

Finalmente, destacaría algunas discusiones que considero importantes para la historiografía centroamericana, relacionada en cómo llamar a los actores políticos: “conservadores o liberales”. Aunque no es reciente esta cuestión para Centroamérica (Bonilla, 1996), si es importante aclararla, por las implicaciones sociales y políticas que tienen estos Secuencia. E-ISSN 2395-8464 
términos políticos en Centroamérica y El Salvador, además de los usos históricos que se le puedan dar. Al respecto la autora optó por diferenciar en "grupos dominantes" y sus actividades en la política para no etiquetarlos ni enmarcarlos en alguna corriente política concreta. Esta es una buena hipótesis de trabajo, pues aporta una flexibilidad en los análisis históricos, especialmente al identificar a personas que no tienen una filiación política e ideológica constante.

Otro tema abordado por la autora consiste en una discusión historiográfica centroamericana, para poner a prueba las teorías del continuismo económico colonial y la teoría de la dependencia para explicar las causas del atraso económico y social de Centroamérica. En su lugar, observa procesos internos y regionales (p. 148) donde resalta a los propietarios de la tierra y comerciantes orientales como responsables del estancamiento y la prolongación del sistema económico que les resultaba benéfico, antes de someter a tensión una larga revisión de procesos que atribuyera el estancamiento a factores externos. Sobre esta propuesta considero que se debe de ahondar todavía más, resaltando otros casos en territorios contiguos como Guatemala, Honduras y Nicaragua durante este mismo periodo. No obstante, esta propuesta es buena para pensar un cambio de paradigma que permita explicar este tipo de fenómenos ocurridos en Centroamérica.

El texto de Pérez Fabregat es un buen comienzo para los interesados en la historia regional de Centroamérica. Además de plantear novedosas interpretaciones a los problemas económicos y sociales de El Salvador, los cuales tienen su origen en el siglo XIX, y las cuales siguen sin ser resueltas, como la violencia y la debilidad institucional de los gobiernos nacionales en Centroamérica. 
RESEÑAS

Referencias

Bonilla Bonilla, A. (1996). The Central American enlightenment 1770-1838: an interpretation of political ideas and political history. San Salvador: FLACSO Programa El Salvador. 